\title{
Stable hybrid myotubes: a new model for studying re-expression of enzymatic activities in vitro
}

\author{
Meola G.*, Sansone V.*, Rotondo G.*, Radice S.*, Bottiroli G.**, Scarlato G.*** \\ * Divisione di Neurologia, Ospedale San Donato, San Donato Milanese \\ ** Centro di Studio per l'Istochimica del CNR, Università di Pavia \\ *** Clinica Neurologica; Centro Dino Ferrari, Università di Milano
}

Heterokaryons represent a stable and reproducible model system for the study of biochemical and molecular aspects responsible for muscle gene activation. Previous experiments have used this fusion system to demonstrate human gene activation in hybrids formed between human and non-human cells. The aim of this research was to apply this experimental model to the correction of a cytoplasmic activity, namely glucose-6-phosphate dehydrogenase (G6PD), in vitro, in hybrid myotubes formed between G6PD-negative and positive myoblasts. Different identification methods were used (Hoechst stain and Fluorescent Latex Microspheres, FLMs) to identify hybrid myotubes formed. We demonstrated the restoration of G6PD activity in all hybrid myotubes formed; we then tried to elucidate the mechanisms underlying the restoration of this specific activity and apply the results obtained to the understanding of more complex mechanisms involved in muscle gene activation.

Key Words: Muscle cell culture - hybrid myotubes - heterokaryons - gene activation - fluorescent latex microspheres - Hoechst stain - glucose-6-phosphate dehydrogenase - myoblast transplantation.

\section{Introduction}

Myoblast cultures provide an advantageous system in which to examine biochemical and molecular changes associated with differentation because myoblast fusion is a naturally occurring process. Cellular differentiation does not appear to be due to loss of genomic material $[6,8,15]$ but rather, to generate and maintain the specialized characteristics of cells of different tissues, genes must be activated and repressed in a well-

Paper presented at the National Congress at Sorrento in 1991 and selected by the Editorial Board of the Journal defined sequential pattern. An elucidation of the mechanisms that regulate changes in gene expression is of central importance to an understanding of the generation and development of more complex cellular systems. When cells of two different species are fused together, either spontaneously or by PEG-induced fusion and chromosome loss and translocation are prevented by culture conditions [3] the fusion product is a stable heterokaryon. Heterokaryons thus represent a stable and reproducible model system for the study of biochemical and molecular aspects responsible for muscle gene activation. Blau et al. [1], in fact, developed this fusion system in this direction: they established hybrids between differentiated muscle cells 
(mouse myotubes) and non-muscle cells (human amniotic cells) and demonstrated that in these stable fusion products, silent human genes were activated. This system also gave the possibility to study whether the products of this gene activation are spatially connected to the nuclei coming from the cell type containing the genetic information. Pavlath et al. [14] have reported that some structural proteins (i.e. human myosin heavy chain and antigen 5.1H11) and a Golgi apparatus enzyme (galactosyl transferase) are only expressed near the nucleus containing the genetic information. They called this restricted region of expression (100 $\mu \mathrm{m})$ a "nuclear domain". However, other authors $[7,12]$ using heterokaryons formed between dystrophin negative and dystrophin positive fibers did not observe a restricted nuclear domain for dystrophin in hybrid myotubes.

Our main objective was to use the hybrid model to study the re-expression of a cytoplasmic activity, namely glucose-6-phosphate dehydrogenase (G6PD), a widely distributed X-linked enzyme, in hybrid myotubes formed between G6PD-deficient and G6PD positive myoblasts. Even though ane$\mathrm{mia}$ is the most frequent clinical manifestation of G6PD deficiency, affecting 400 million people worldwide, a muscular form of this deficiency has been decribed [4]. We thus prepared cell cultures from muscle biopsy specimens of patients affected by the G6PD Mediterranean variant, one of the most common, which has the advantage of expressing the defect in vitro (compared to other variants where the enzymatic activity is not always detectable) and the restoration of G6PD activity in this fusion system was examined in a variety of ways. The next step was elucidate the mechanisms underlying the restoration of this specific activity and apply the results obtained to the understanding of more complex mechanisms involved in muscle gene activation.

\section{Material and method}

The potential to restore a cytoplasmic activity like G6PD in hybrid myotubes formed between enzyme deficient and normal cell types was investigated in a variety of ways. The hybrid model proved to be a stable fusion system for studying the mechanisms involved in the restoration of G6PD activity in the fusion products obtained.

Normal human myoblasts were obtained from muscle biopsy specimens from patients who were ultimately deemed to be free of muscle disease and G6PD-deficient human myoblasts were obtained from the patient muscle specimens with appropriate ethical approval. Mass muscle cultures in pre-fusion (mononuclear cells) and postfusion stages (myotubes) from normal and defi-

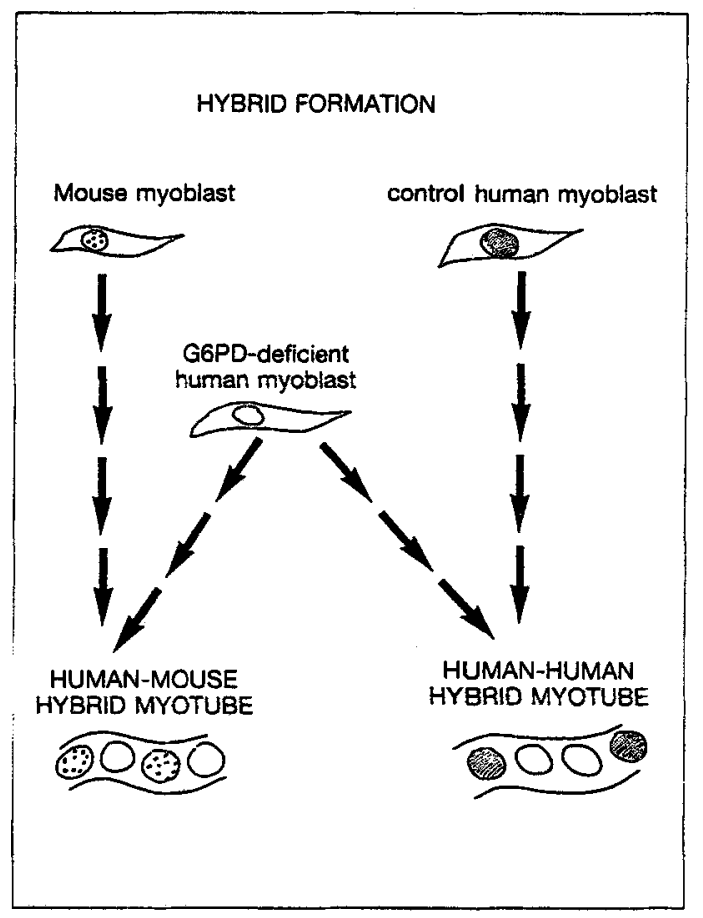

Fig. 1. Hybrid formation.

cient patients were established according to previously described methods [10].

Human myoblasts were grown in proliferative medium (Muscle Medium: $\mathbf{M M}=$ Minimum Essential Medium - MEM - Earle's Salts enriched with non-essential aminoacids, pyruvate, vitamins in the presence of $75 \%$ Units/ml penicillin, $75 \mathrm{ug} / \mathrm{ml}$ streptomycin and $0.25 \mathrm{ug} / \mathrm{ml} \mathrm{am-}$ photericin B) enriched with FCS $13 \%$.

Mouse myoblasts were obtained by a dissociation method using 15-16 day old fetal mouse muscle (HaM/ICR) as previously described. Wistar adult mice (80-120 gr.) obtained from Charles River Labs were used in all studies.

Hybrid formation: about 25.000 G6PD-deficient human myoblasts were harvested and mixed with 25.000 mouse myoblasts (grown in a specific medium: M199 added with CEE and HS $15 \%$ ) or with 25.000 normal human myoblasts so that a $1: 1$ ratio of normal myoblasts and G6PD-deficient myoblasts was obtained (Fig. 1). In other trials different cell densities were used so that the nuclear ratio in the hybrids formed could be more easily manipulated. The cell mixture was then plated on glass cover-sides in $24 \mathrm{~mm}$ multi-well Costars.

Hybrid myotubes were obtained by spontaneous fusion or by polyethylene glycol (PEG) induced fusion. PEG 1000 mol. wt. was used; to prepare 

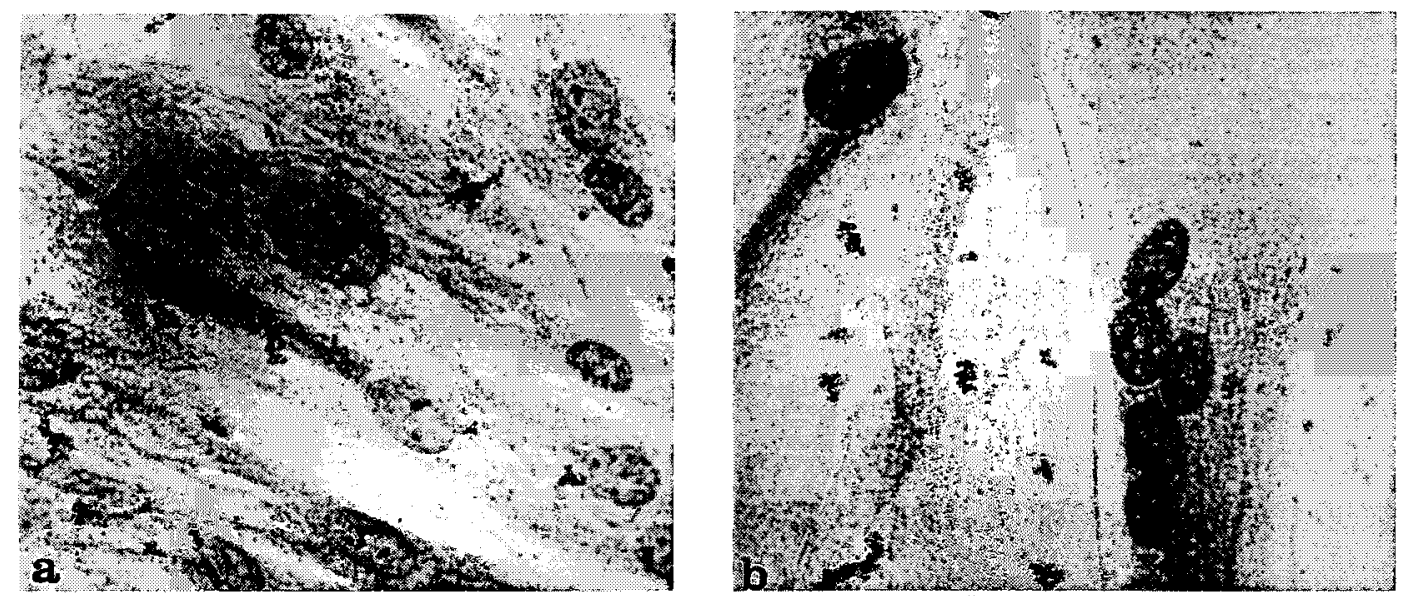

Fig. 2. Fibroblasts treated with PEG 1000. a: mononucleated fibroblasts are present in a monolayer in the area examined BEFORE PEG treatment. b: same culture AFTER I minute treatment with PEG 1000. The cells were observed and photographed after 48 hours in culture with the appropriate medium.

PEG solution, PEG was autoclaved and warm MM medium (without serum) was added to the warm PEG to obtain a $50 \%$ solution. The concentration of the PEG solution is expressed on a weight/ weighs basis, assuming that $1 \mathrm{ml}$ of medium weights $1 \mathrm{gr}$. The fusion protocol adopted is as previously described [5]. A differentiation medium containing MEM with 5\% HS [10] was then used and changed each day until hybrid formation occurred. Histochemical and biochemical analysis were then carried out. To demonstrate that PEG actually induced fusion and that this did not affect the vitality of the cells in culture, fusion was PEG-induced in fibroblasts which normally do not form multinucleated cells (Fig. 2).

G6PD staining: the histochemical reaction was performed on the patient muscle cultures, on normal human and deficient myoblasts and myotubes and on the hybrid myotubes obtained. For a cytochemical demonstration of G6PD, muscle cultures were rinsed in Earle's balanced salt solution (EBSS), drained and air dried. The cells were then stained for G6PD activity by incubating for 50 minutes at $37^{\circ} \mathrm{C}$, in a specific medium containing $100 \mathrm{mM}$ G6P, $1 \mathrm{mM}$ MgC12, $0.6 \mathrm{mM}$ Nitroblue Tetrazolium (NBT), $100 \mathrm{mg} / 10 \mathrm{ml}$ polyvinylpyrrolidone in $0.2 \mathrm{M}$ Tris buffer $\mathrm{pH} 7.8$. Background reaction was performed without substrate G6P. G6PD histochemical activity was observed with Zeiss microscope. G6PD staining was compared in short- and long-term cultures to evaluate the time-course of this enzymatic activity.

Hoechst 33258 staining: (Sigma, St-Louis, USA). This is a DNA - specific dye used to distinguish the hybrid myotubes formed between human and mouse myoblasts according to their fluorescent pattern: the human nuclei were smooth and the mouse nuclei were dotted.

When enough myotubes were present (more than 100 ), the cultures were stained for 10 minutes with Hoechst $(10 \mu \mathrm{g} / \mathrm{ml})$. Then a coverslip was placed over a thin layer of $50 \%$ glycerol solution.

Biochemical investigation: for biochemical investigation, cultures were rinsed with cold $10 \mu \mathrm{M}$ NADP buffer and harvested by trypsinization. The enzymatic assays were performed spectrophotometrically on cell suspensions according to the described method [11].

Fluorescent Latex Microspheres (FLM): it is possible to verify the formation of hybrid myotubes between G6PD-deficient and normal human myoblasts using Fluorescent Latex Microspheres (FLM) (Luma Fluor, New City, New York). Two types of microspheres (rhodamine and fluorescein spheres) were incorporated in the two different myogenic cell lines for 12 hours at a dilution of 1:2500 in Muscle Medium enriched with 5\% FCS. Cells from the two myogenic cell lines were then mixed at a ratio of 1:1 and grown in a differentiating medium (M199+5\% HS, penicillin, streptomycin and glutamine). Cell cultures were then observed with a fluorescent microscope until myotube formation occurred. The formation of hybrid myotubes was demonstrated by the presence of both types of microspheres within the same myotube [9]. 

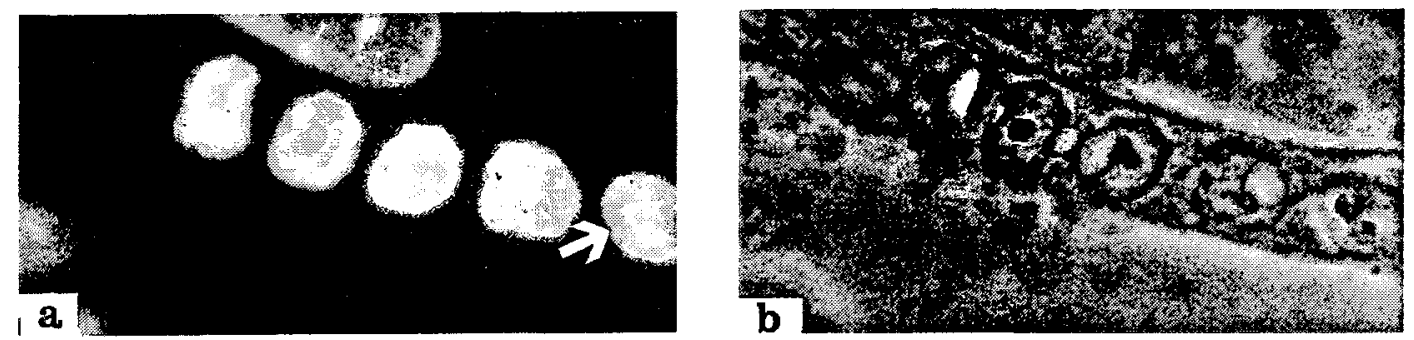

Fig. 3. The use of Hoechst stain to distinguish human from murine nuclei. a: Hoechst stain gives a dotted appearance to the murine nucleus (arrow); human nuclei instead (4 in the picture) appear uniformly stained. b: same myotube in phase-contrast microscopy.
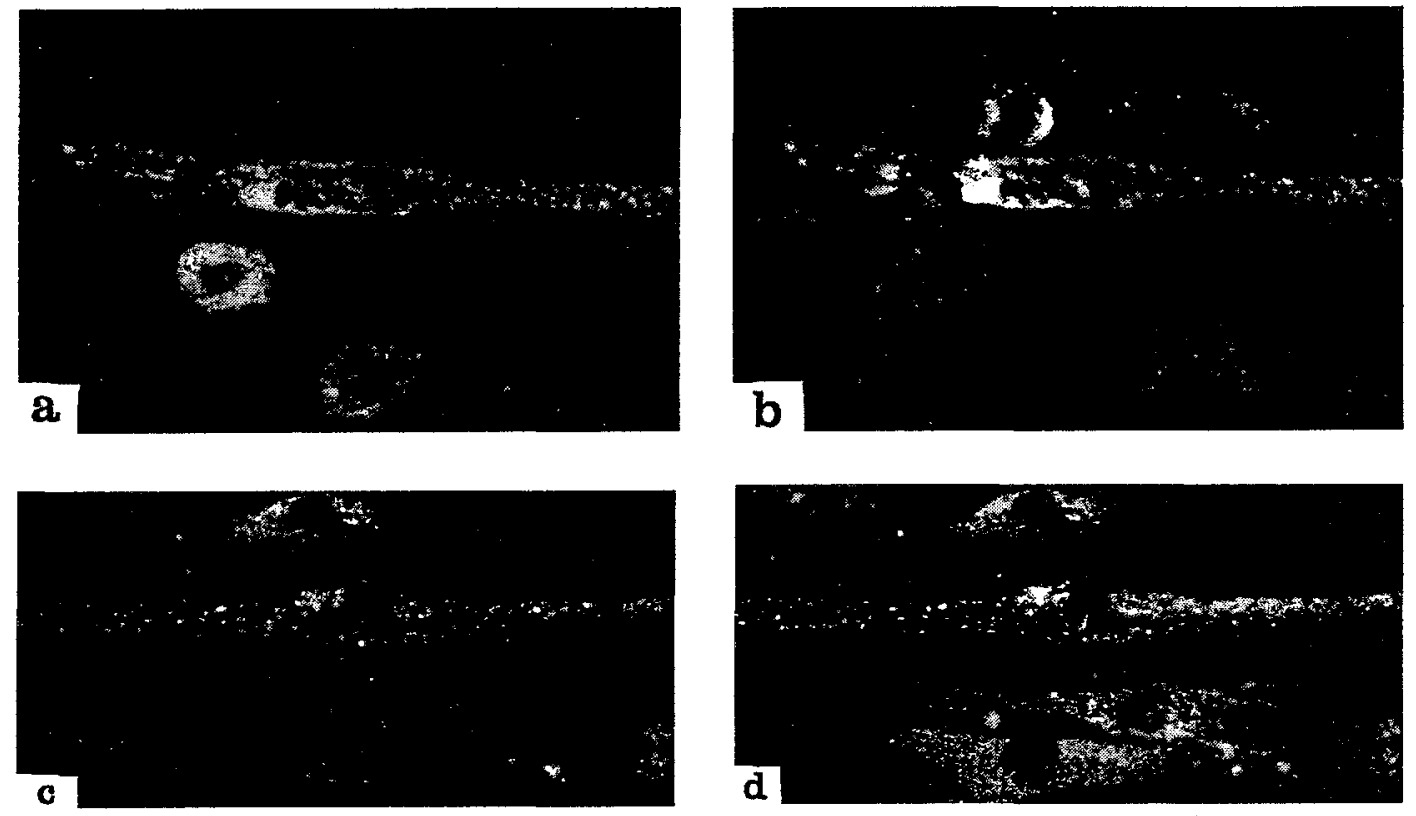

Fig. 4. Fluorescent latex microspheres (FLMs). a: rhodamine and b: fluorescine coloured myotube after 5 weeks in culture. c: rhodamine and d: fluorescine myotube after 6 weeks in culture.

\section{Results}

Our first objective was to optimize cellular fusion using PEG and to identify hybrid formation by the use of different methods. PEG actually proved to act on cell membranes because treatment on normally mononucleate cells (fibroblasts) just like treatment on myoblasts effectively induced fusion in the fibroblasts tested for control (Fig. 2A and 2B). Moreover it did not seem to affect the viability of the cells in culture and histochemical and staining reactions could be carried out as usual. Similar results were obtained in hybrids produced by spontaneous and PEG-induced fusion.

The first identification method using Hoechst stain proved to be quite effective in distinguishing mouse and human nuclei because, as has been previously demonstrated [14], mouse nuclei appear dotted compared to the uniform distribution of the stain in human nuclei (Fig. 3A and 3B). However this method has the disadvantage that it implies fixation. Different nuclear ratios were obtained in the human-mouse hybrids formed but this did not appear to be connected to the cell densities used because the nuclei predominating in the hybrid formed were not necessarily the ones belonging to the cell type plated at higher densities. By varying the densities at which mixture occurred hybrid myotubes were also obtained at ratios other than 1:1.

The second identification method using Fluorescent Latex Microspheres (FLM) of different co- 

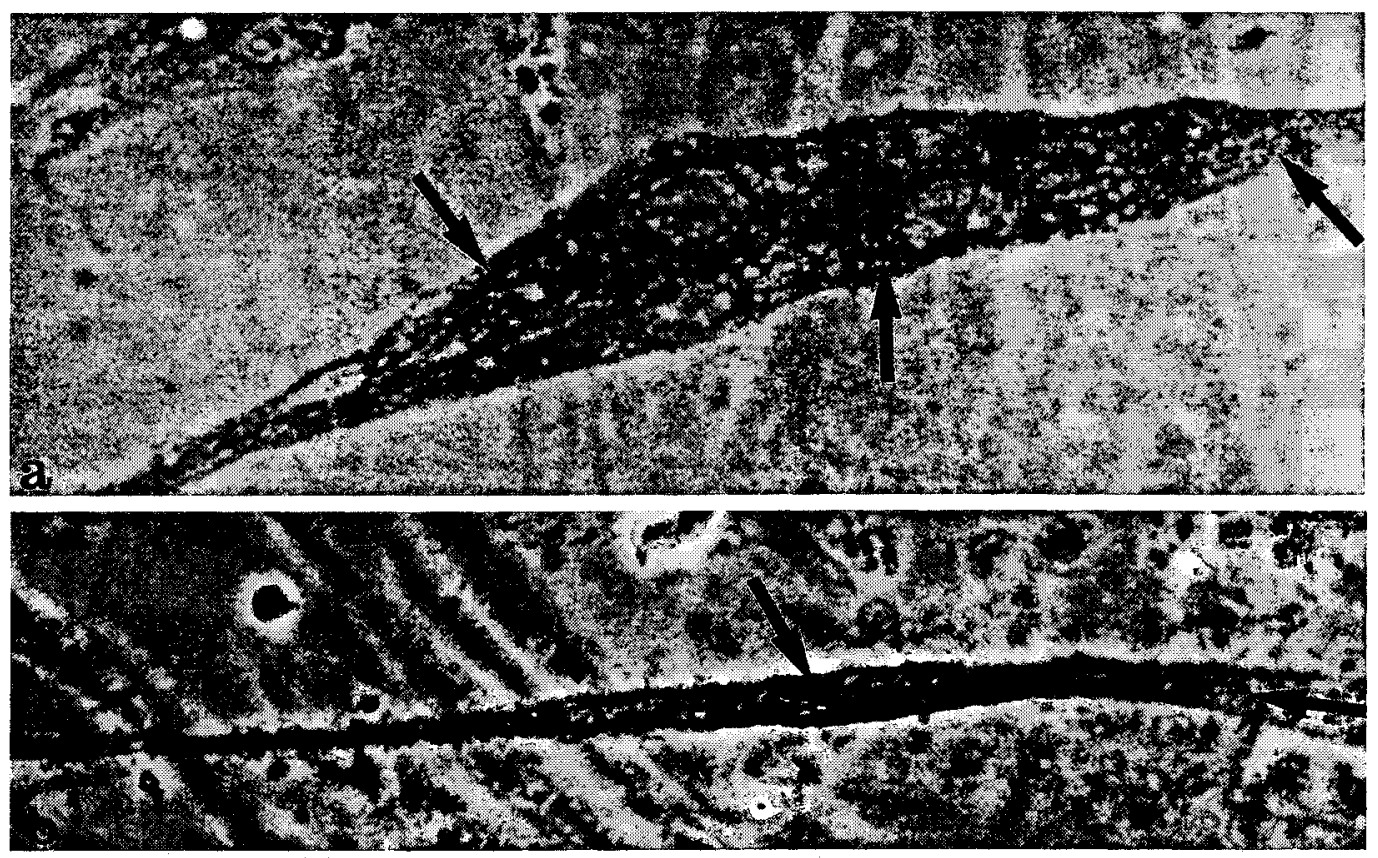

Fig. 5. G6PD activity in long-term cultures. a: G6PD granules can be seen uniformly distributed throughout the human-human hybrid myotube after 8 weeks in culture (arrows). b: similar granular appearance after 9 weeks in culture (arrows) in a human-human hybrid myotube.

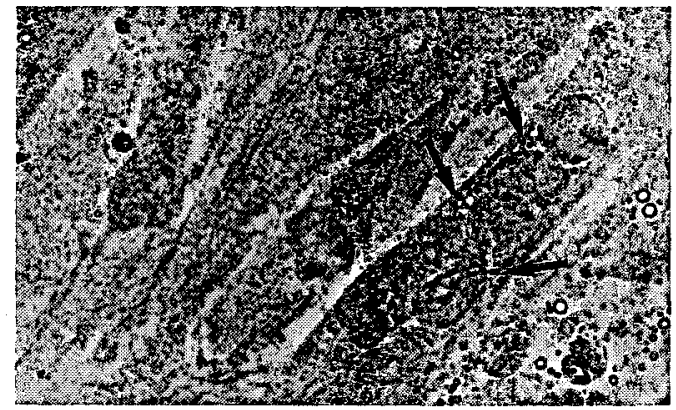

Fig. 6. G6PD-deficient human myotube. Note the decrease in the number and distribution (arrows) of G6PD granules throughout the myotube.

lors has the advantage that the presence of a hybrid myotube can be detected in a living culture by observation with a fluorescent microscope but has the disadvantage that the position and the identification of the competent nuclei cannot be recognized. Our results confirmed that these fluorescent latex microspheres do not affect fusion, proliferation or viability of the cells in culture [9]. We were also able to observe fluorescence easily after 5 or 6 weeks in culture (Fig. 4A and 4B and $4 \mathrm{C}$ and $4 \mathrm{D}$ respectively). It was then also possi- ble to test for G6PD activity in long-term cultures (up to 8-9 weeks in culture) to verify the stability of the specific restoration and demonstrate that this fusion system also has the advantage that the experimental investigations can be carried out in vitro in long-term cultures (Fig. 5A and 5B).

The next step was to evaluate G6PD activity in the myotubes obtained from our patients. In confirmation of our previous observations [4] the defect in the Mediterranean variant is expressed in vitro (Fig. 6). Our main objective here was to verify whether there was a correction of this lack of enzymatic activity in the hybrid myotubes. Our results indicate that there is a restoration of G6PD activity in the hybrid myotubes (Fig. 7A) formed spontaneously between normal and G6PD-deficient human (Fig. 6) myoblasts and between normal murine and G6PD-deficient myoblasts (Fig. 6). Similar results were also obtained following PEG induced fusion.

In all hybrid myotubes obtained, G6PD granules reappeared throughout the cytoplasm although this activity was less pronounced in human-mouse hybrid myotubes (Fig. 8A) than in human-human hybrids (Fig. 7A).

Human-human hybrid formation was confirmed by the presence of both types of microspheres within the same myotube (Fig. 7B and 7C) and humanmouse hybrid formation by the presence of dotted 

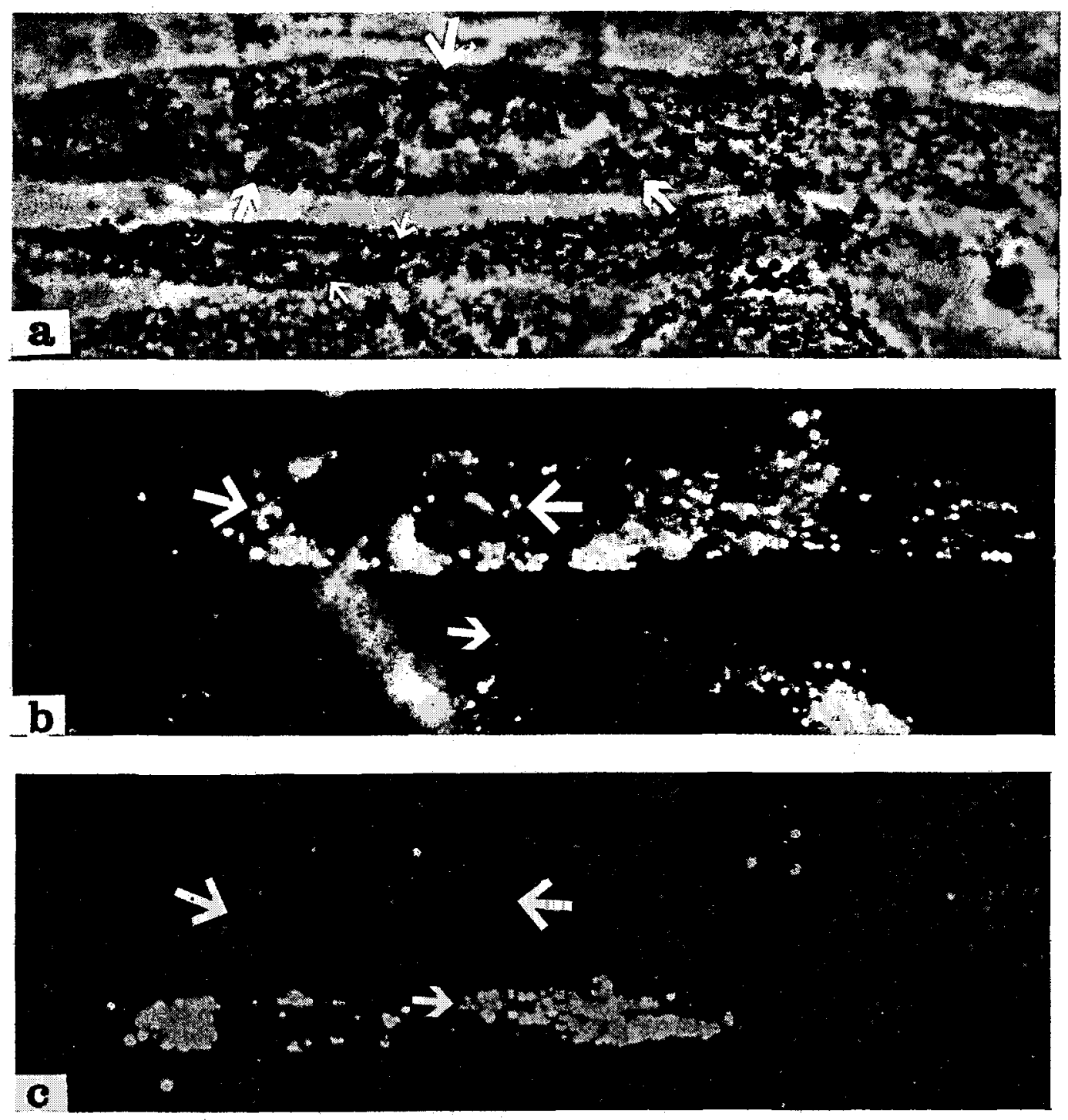

Fig. 7. G6PD deficient- normal human hybrid myotubes. a: restoration of G6PD activity in the human-human hybrid myotube obtained by spontaneous fusion ( 2 weeks in culture). This hybrid myotube was formed by the fusion of normal human myoblasts (labelled with rhodamine FLMs) and G6PD deficient human myoblasts (labelled with fluorescein FLMs). The same hybrid myotube as in a observed with a fluorescent microscope and tested for the presence of fluorescein (b) and rhodamine (c) microspheres to confirm hybrid formation. The arrows indicate the presence of both types of FLMs in the same region of the myotube.

and uniformly stained nuclei using Hoechst stain in the same myotube (8B); Hoechst staining was employed to verify whether restoration of enzymatic activity was dependent on the position of the competent nucleus, as has been previously described in muscle-non muscle heterokaryons [3, 13]. Our data clearly indicate that the re-expression of enzymatic activity is independent of the position of "competent" nuclei (Figure 9A, 9B, 9C and 9D). In all human-mouse hybrid myotubes obtained G6PD activity appears uniformly distributed throughout the cytoplasm, irrespective of different nuclear ratios. G6PD staining did not interfere with any of the identification methods used. 

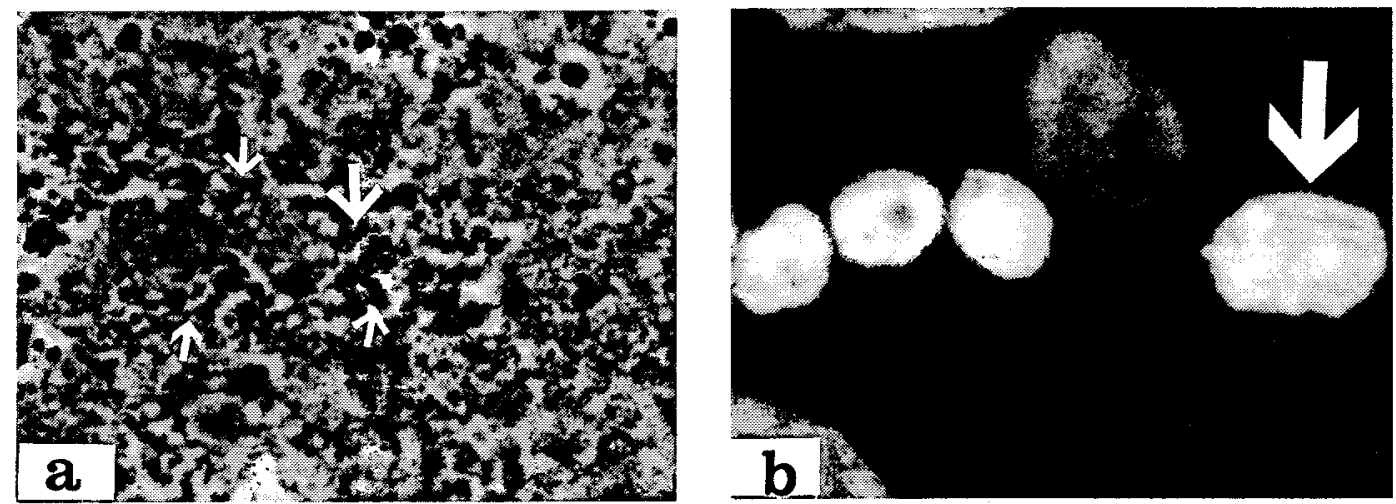

Fig. 8. Hybrid myotube formed by human G6PD-deficient and murine myoblasts. a: human-mouse hybrid myotube: the restoration of diffuse enzymatic activity (arrow) is clearly evident. The same myotube observed after Hoechst staining in b to confirm hybrid formation: note the presence of 1 murine dotted nucleus (arrow) and 3 human nuclei (smooth appearance).

\section{Discussion}

Heterokaryon experiments suggested that the process of cell specialization is not fixed, i.e. heterokaryon facilitate "genetic approach" to the iso- lation of the genes encoding factors that intervene at different steps in the muscle regulatory hierarchy [2].

Activation was detected for genes encoding different muscle functions: an enzyme creatine-kin-
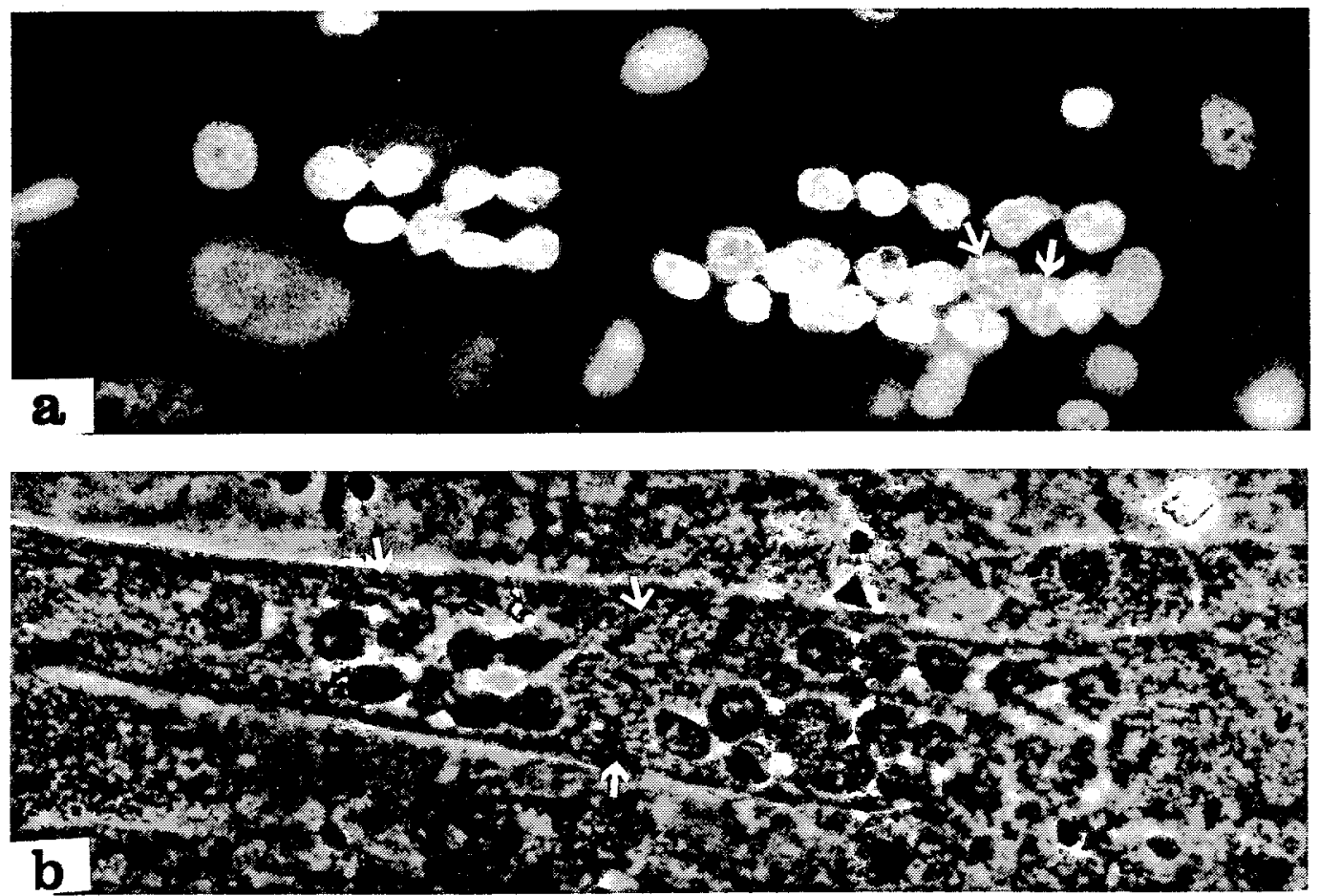

Fig. 9. Evaluation of G6PD activity in hybrid myotubes formed between G6PD-deficient and murine myoblasts. a. myotube observed after Hoechst staining showing 2 murine nuclei (arrows) and 26 human nuclei and b after G6PD staining after 2 weeks in culture. Notice the initial distribution of enzymatic activity (arrows) in this large myotube. 

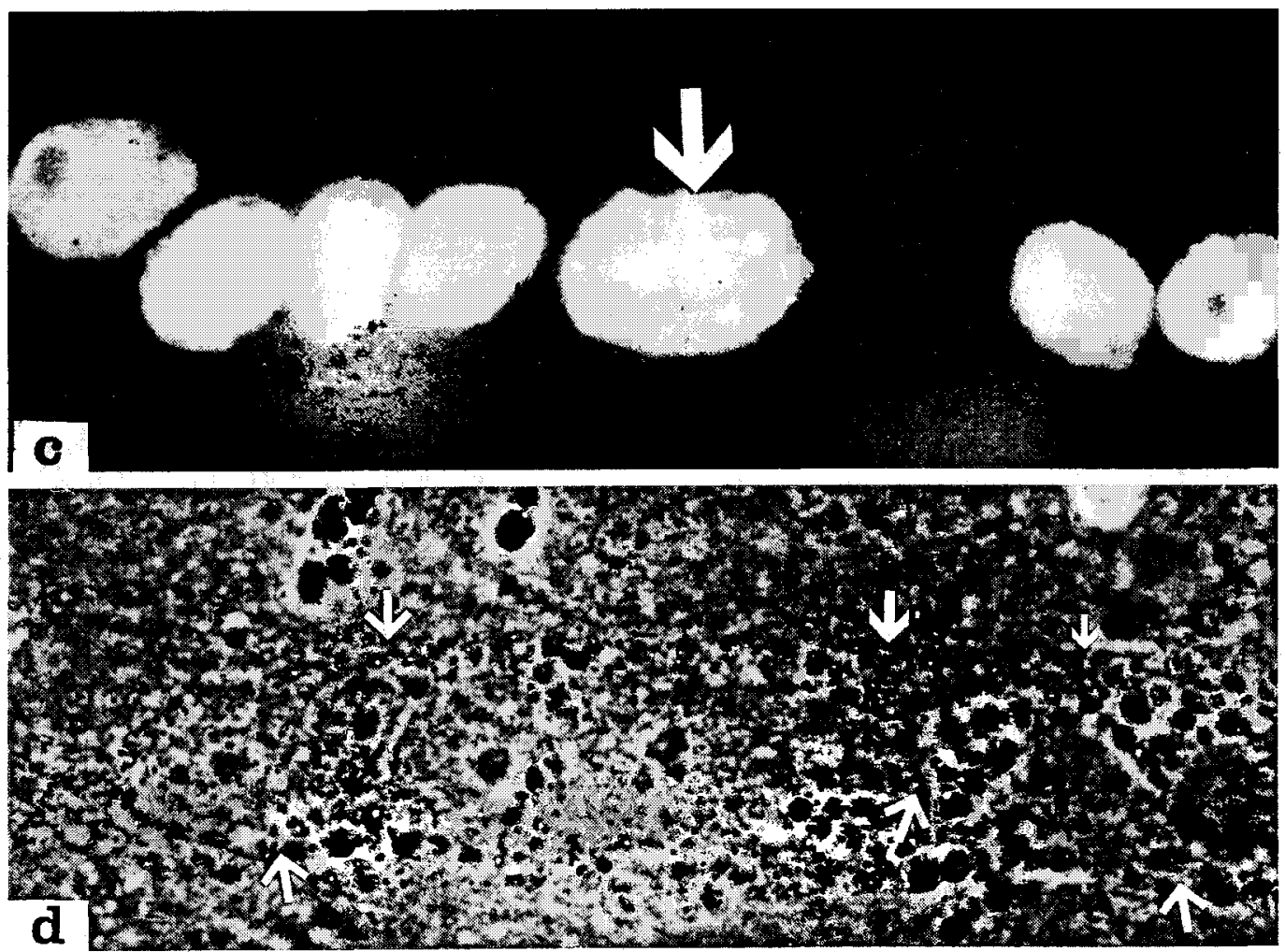

Fig. 9. c: myotube with I murine nucleus (arrow) and 6. human nuclei. d: the same myotube in-which G6PD granules are seen throughout the myotube (arrows). Note the restoration of G6PD activity is independent of the position of the competent nuclei.

ase; two cell-surface antigens 51H11 and 24ID5 and components of the contractile apparatus myosin light-chains, myosin heavy-chains, sarcomeric actin transcript. All these previous experiments confirmed the plasticity of the differentiated state of muscle where genes are switched on and off giving rise to different phenotypes.

This is, to our knowledge, the first application of a heterokaryon system formed between G6PD-negative and G6PD-positive myoblasts in the attempt to restore the enzymatic activity in the newly-formed myotubes.

The identification methods used to recognize hybrid myotubes, proved to be extremely effective essentially because it was possible to obtain different information. FLMs were used not only to identify hybrids but also to test for G6PD activity in long-term cultures. In fact the detectability of both colored microspheres after 6 weeks in culture allowed us to obtain data concerning the time-course of this enzymatic activity in the hybrid myotubes formed: G6PD granules can still be detected after 8 and 9 weeks in culture throughout the cytoplasm; this is important because this hybrid model proves to be an adequate in vitro sys- tem for studying cytoplasmic activities like G6PD. Hoechst stain instead enabled us to evaluate whether diffusible molecules that are transported through the cytoplasm, mediate the activation of genes observed in heterokaryons, since the nuclei of the two cell types remain separate and distinct. Results obtained using Hoechst stain indicate that regardless of the number and position of the "competent" nuclei, the distribution of granules was similar in all hybrid myotubes observed. One normal nucleus is sufficient to restore enzymatic activity throughout the hybrid myotube and granules are detected throughout the cytoplasm and not only in the area immediately surrounding the competent nucleus. This suggests that G6PD reexpression could be due to a "passive" distribution of enzyme activity throughout the hybrid myotube formed.

This experimental model thus proves to be an adequate one for studying the expression of this enzymatic activity because: i) the defect is expressed in vitro; ii) it is corrected in all hybrid myotubes obtained and iii) the restoration is maintained in long-term cultures. This not only provides the scientific background for myoblast 
transfer therapy but suggests that the correction obtained when competent and deficient nuclei intermingle in the cytoplasm of heterokaryons is not a transitory event. The restoration of G6PD activity demonstrates that this fusion system proves to be an adequate experimental model not only for elucidating the specific mechanisms involved in G6PD re-expression but also for clarifying the molecular aspects of regulation of gene expression.

\section{Sommario}

Il modello degli ibridi costituisce un sistema valido e riproducibile per studiare gli aspetti biochimici $e$ molecolari implicati nell'attivazione genica. Tale sistema di fusione è stato utilizzato da precedenti autori per dimostrare l'attivazione di specifici geni umani in ibridi formati dalla fusione di cellule umane con cellule non-umane. Lo scopo di questa ricerca è stato quello di applicare il modello sperimentale degli ibridi per valutare la correzione di un'attività citoplasmatica, quale la glucosio-6-fosfato deidrogenasi (G6PD), in vitro, in ibridi formati tra mioblasti G6PD-deficitari e normali. Sono stati impiegati diverse metodiche per identificare $i$ miotubi ibridi (colorante nucleo-specifico, Hoechst e microsfere di lattice fuorescinate e rodaminate). I nostri risultati indicano che vi è un ripristino dell'attività G6PD in tutti $i$ miotubi ibridi formati; abbiamo quindi tentato di comprendere i meccanismi specifici sottostanti alla ricomparsa di quest'attività enzimatica per poterli applicare alla comprensione dei più complessi meccanismi implicati nell'attivazione di geni muscolari.

Address reprint requests to:

Prof. Giovanni Meola

Divisione di Neurologia

Ospedale San Donato

Via Morandi 30

20097 San Donato Milanese (MI)

\section{References}

[1] Blau H.M., Chiu C.P., Webster C.: Cytoplasmic activation of human nuclear genes in stable heterokaryons. Cell 32:1171-1180, 1983.

[2] BLAU H.M.: Hierarchies of regulatory genes may specify mammalian development. Cell 53:673, 1988.

[3] Blau H.M., Pavlath G.K., ChoY-Pik ChiU ET AL.: Heterokaryons: a model system for studying cell commitment and differentiation. Cellular and Molecular Biology of Muscle Development, pages Alan R. Liss, Inc. 851-861, 1989.

[4] Bresolin N., Bet L., Moggio M., Meola G. ET AL.: Muscle glucose-6-phosphate dehydrogenase deficiency. J. Neurol. 236:193-198, 1989.

[5] Davidson R.L., O'Malley K.A., Wheeler T.B.: Polyethylene Glycol-induced mammalian cell hybridization: effect of Polyethylene Glycol molecular weight and concentration. Somatic Cell Genetics, vol. 2,3:271-280, 1976.

[6] DE ROBERTIS E.M., GORDON J.B.: Gene activation in somatic nuclei after injection into amphibian oocytes. Proc. Natl. Acad. Sci. 74:2470-2474, 1977.

[7] Gearhart J.D., Mintz B.: Clonal origins of somites and their muscle derivatives; evidence from allo phenic mice. Develop. Biol. 29:27, 1972.

[8] GORDON J.B.: The developmental capacity of nu- clei taken from intestinal epithelium cells of feeding tadpoles. J. Embryol. Exp. Morphol. 10:622$640,1962$.

[9] LabrecQue C., Huard J., Dansereau G. et al.: A new technique to identify hybrid myotubes in vitro without culture fixation. J. Histochem. Cytochem. 39:139-143, 1991.

[10] Meola G., Scarpini E., Velicogna M. et al.: Cytogenetic analysis and muscle differentiation in a girl with severe muscular dystrophy. J. Neurol. 233:168-170, 1986.

[11] Meola G., Bresolin N., Rimoldi M. et al.: Re cessive carmitine palmityl transferase deficiency: biochemical studies in tissue culture and platelets. J. Neurol. 235:74-79, 1987.

[12] PARTRIDGe T.A., SLOPER J.C.: Evidence of fusion between host and donor myoblasts in skeletal muscle grafts. Nature 237:306-308, 1978.

[13] PAVLATH G.K., BLAU H.M.: Expression of muscle genes in heterokaryons depends on gene dosage. J. Cell. Biol. 102:124-130, 1986.

[14] Pavlath G.K., Rich K., Webster S.G., Blau H.M.: Localization of muscle gene products in nuclear domains. Nature 337:570-573, 1989.

[15] SCHMID V., ALDER H.: Isolated mononucleated, striated muscle can undergo pluripotent transdifferentiation and form a complex regenerate. Cell $38: 801-809,1984$ 